\title{
Linearization of an Outphasing Amplifier for Wide-band Multi-carrier Signals
}

\author{
Keith Finnerty, Robin Wesson, John Dooley, Mustafa Acar, Mark P. van der Heijden, and Ronan Farrell
}

\begin{abstract}
A novel method using self-organizing maps to determine the optimum ranges to partition the vector switched Volterra series is presented. Analyzing characteristics of the input signal it is possible to separate different regions of operation. Partitioning of the correction algorithm provides the ability to target regions of different non-linear behavior with separate coefficients. This work is validated with experimentally measured results and shows an adjacent channel power ratio (ACPR) of $-49 /-50 \mathrm{dBc}$ achieved for a four carrier Wideband Code-Division Multiple Access (WCDMA) signal using a 19 watt digital outphasing amplifier from NXP.
\end{abstract}

Index Terms - Linear amplification with non-linear Components (LINC), linearization, outphasing amplifier, predistortion.

\section{INTRODUCTION}

$\mathbf{T}$ HE power amplifier (PA) can consume the largest amount of power of any single component in the base station radio frequency transceiver. As a result the efficiency with which they operate greatly impacts system efficiency and cooling requirements. This is a fundamental issue and has driven the investigation of higher efficiency amplifier architectures such as Doherty, envelope tracking (ET) and outphasing PAs. The digital outphasing amplifier topology achieves increased efficiency through load modulation. A pair of constant envelope signals are amplified by identical amplifier stages, the recombined signal reconstructs the desired amplitude modulated signal, counteracting the nonlinear effects associated with switch mode operation. These benefits come at a cost, the two path topology of the amplifier has alternative requirements for linear operation; key amongst those is phase, gain and delay imbalance between the amplifier paths. Additional amplitude and phase distortion introduced by the recombination stage leads to non-ideal operation. In recent years many of these problems have been resolved to some degree. In [1] the author extracts both amplitude and phase distortion generated from an ideal Chireix combiner, from this a predistortion function is derived for the input signal. In [2]

Manuscript received July 29, 2014; revised October 24, 2014; accepted November 15, 2014. Date of publication January 09, 2015; date of current version February 10, 2015. This work was supported by Science Foundation Irelandunder Grant $10 / \mathrm{CE} / \mathrm{I} 1853$ and by HEA under PRTLI5 and is being co-funded by the Irish Government and the EU under Ireland's Structural Funds Programmes 2007-2013: Investing in your future.

K. Finnerty, J. Dooley, and R. Farrell are with the Callan Institute, Department of Electronic Engineering, National Institute of Ireland, Maynooth, Kildare, Ireland (e-mail: kfinnerty@eeng.nuim.ie).

R. Wesson, M. P. van der Heijden, and M. Acar are with NXP Semiconductors, Eindhoven $5656 \mathrm{AE}$, The Netherlands (e-mail: robin.wesson@nxp.com).

Digital Object Identifier 10.1109/LMWC.2014.2382653

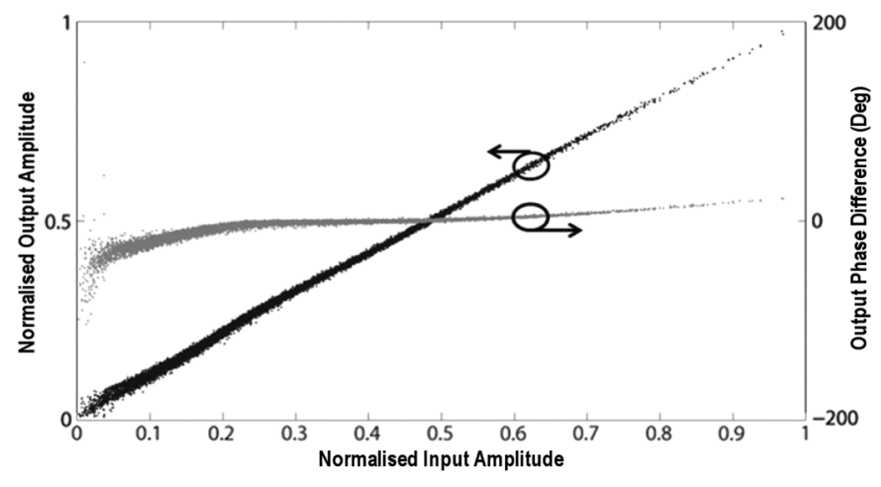

Fig. 1. AMAM/AMPM of digital outphasing amplifier - The outphasing function is a custom LUT.

a proposed linearization method modifies the phase of the outphasing signals using a look up table approach, the correction is a function of input amplitude and frequency. In [3] a phase polynomial for each outphasing path is derived, correcting amplitude and phase distortion of the output signal. This method is then further improved in [4]with a more complex model for the outphasing amplifier including the phase polynomial as well as amplitude and path delay effects. These methods have greatly improved the linear PA performance however wide band operation still remains a challenge for base station specifications.

The bandwidth expansion of an outphasing signal is typically 10-12 times the bandwidth of the desired transmission signal [5]. The outphasing topology requires identical hardware in each of the signal paths, for wideband operation this can be difficult to achieve within a discrete setup. Statically we calibrate the system across the operating frequency, providing the outphasing amplifier with balanced input signal paths. Removing the imbalance effects allows the system to be characterized and linearized as a single input-output amplifier system. Since this calibration procedure is performed statically it cannot account for additional imbalances that can occur during dynamic operation. The result is spreading in the output signal, appearing similar to memory effects seen in traditional amplifier architectures, which can be seen in Fig. 1.

In Fig. 1 the region of AMAM plot with the largest deviation from ideal behavior occurs at lower powers as a result of outphasing amplifier difficulty to achieve a null. Non-Ideal effects in the outphasing amplifier are caused by dynamic operation of the amplifier and path mismatch such as amplitude and phase imbalance and dc offset. It is visible in Fig. 1 that there are relatively large deviations from ideal behavior at low powers. If a large time series is used to train DPD more emphasis is placed 
on the operation of the PA at higher powers. To avoid this a segmented approach such as that used in [6] and [7]is used where equal consideration can be given to train each of the separate regions of operation. In this letter we demonstrate a hardware implementation of a single input-output correction, enabling wide band operation of an outphasing amplifier to meet 3GPP ACPR specifications. This work follows on from the linearization of single carrier WCDMA signal presented in [6], as signal bandwidth increases linearization becomes more challenging. Investigated in this letter is a 19 watt $\mathrm{GaN}$ digital outphasing amplifier developed at NXP semiconductors [8].

\section{Vector SWitched VolterRa Using SELF ORGANIZING MAPS}

The Volterra series is a powerful time series capable of non linear system modeling. It has been widely used in PA behavioral modeling and linearization. The equation for the Volterra series is outlined in (1) where $\tilde{x}(n)$ and $\tilde{y}(n)$ are the input and output complex envelope signal samples, $\tilde{h}_{p}\left(i_{l}, \ldots, i_{p}\right)$ denotes the discrete time Volterra kernels. $N L$ is the order of nonlinearity, $M$ is the memory depth and ${ }^{*}$ denotes the conjugate transpose.

$$
\begin{array}{r}
\tilde{y}(n)=\sum_{p=1}^{N L} \sum_{i_{1}=0}^{M} \ldots \sum_{\substack{i_{p+1}=\frac{i_{p-1}}{2} \\
(p+1) / 2}}^{M} \sum_{\frac{i_{p+3}}{2}=0}^{M} \ldots \sum_{i_{p}=i_{p-1}}^{M} \tilde{h}_{p}\left(i_{1}, \ldots, i_{p}\right) \\
\prod_{r=1}^{p} \tilde{x}\left(n-i_{j}\right) \prod_{j=\frac{(p+3)}{2}}^{p} \tilde{x}^{*}\left(n-i_{j}\right) \quad(1)
\end{array}
$$

An advancement on this is segmented or piecewise Volterra algorithms. The Vector switch approach uses not only the current input amplitude, $\operatorname{mag}(X(t))$, but also time delayed with a time delay, $\operatorname{mag}(X(t-n))$ to generate a vector space. This vector space is then quantized using a clustering algorithm, resulting in unique subdivisions of the input data. To each subdivision an individual time series is applied, in our case we choose Volterra series. The result is a more robust algorithm and in many cases a more efficient implementation as each subdivision requires fewer coefficients. In [7]the author uses K-means to cluster the input data, an efficient algorithm to train with a simple code book or look up table (LUT) implementation.

$$
\begin{aligned}
S(t) & =A(t) e^{-j 2 \pi \varphi(t)} \\
\Delta \varphi(t) & =\text { unwrap }(\varphi(t)-\varphi(t-n))
\end{aligned}
$$

In this letter we examine additional parameters of the input signal to improve linearization of the outphasing amplifier. As described in the introduction the majority of the non-linearity for the amplifier resides at lower output powers, in particular around the zero crossing point. For a complex modulated signal the zero crossing point occurs as the signal passes through the centre of the unit circle. This also corresponds to a rapid change in signal phase. Utilizing the rate of change of phase it is possible to extract additional information from the input signal, increasing the number of clusters at lower signal power levels. Equations (2) and (3) show the method of calculating a delta phase value where $\varphi(t)$ is the phase of the current input signal $S(t), \varphi(t-n)$ is the phase of time delayed sample and $\mathrm{A}(\mathrm{t})$ is the

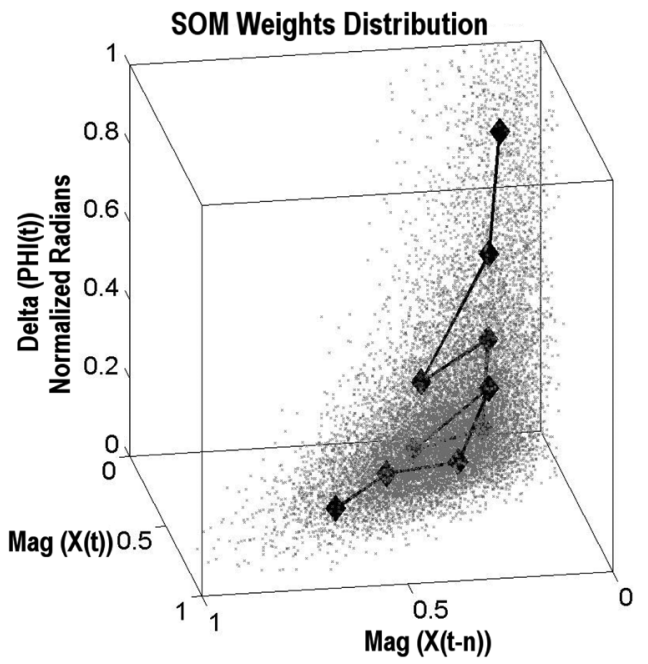

Fig. 2. Self Organizing Map (SOM) weight locations (Black), input data (Grey). In this example $n=3$.

amplitude modulation component of the input signal. The unwrap function is used to remove discontinuities associated with phase as it rotates about the unit circle.

Investigation into multi dimensional clustering algorithms outlined some possible issues using K-means in practice. As the number of dimensions increase the code book or LUT for K-means will increase exponentially, possibly impacting the practicality of the hardware implementation. Self organizing maps (SOM) offer an alternative implementation, derived from a special case of artificial neural network that is used to produce a discrete representation of the input space, referred to as a map. SOM is implemented using an array of adders and multipliers as well as a traditional cluster LUT method it offers a more compact solution as the number of inputs grow. SOM presents a more robust method for clustering compared with K-means [9]. Fig. 2 illustrates the 3 dimensional mapping process proposed in this letter for outphasing amplifiers. This increase in robustness is due to the training method, each input sample effects all neurons not just the closest or winning neuron. The learning rate associated with each neuron is inversely proportional to its distance to the neuron that is closest to the input sample. As a result the network is more robust to the problem of individual neuron local minimum. The main disadvantage with SOM is the increased computational complexity of the training algorithm. The increase in the computational complexity for a SOM with ten output clusters is twenty times [10]. A dramatic increase, however the robustness of the SOM requires fewer iterations to reach as good or better results. In this example SOM ran for a total of 25 iterations, $25 \%$ fewer than the K-means algorithm required. Fig. 2 presents an example of three dimensional clustering of parameters extracted from the input signal. The parameters of the input signal are $\operatorname{mag}(X(t)), \operatorname{mag}(X(t-n))$ as well as the additional $\Delta \varphi(t)$ parameter. A total of ten individual clusters are extracted.

\section{ExPERIMENTALly MeAsured Results}

The small signal system is calibrated initially to remove quadrature imbalance, local oscillator feed through, amplitude 
TABLE I

PERFoRMANCE COMPARISON OF LINEARIZATION TECHNIQUES

\begin{tabular}{lcc}
\hline \hline $\begin{array}{l}\text { Linearization } \\
\text { Technique }\end{array}$ & $\begin{array}{c}\text { Adjacent } \\
\text { ACPR }\end{array}$ & $\begin{array}{c}\text { Maximum } \\
\text { output power }\end{array}$ \\
\hline \hline None & $\mathbf{- 2 6 d B c}$ & $18 \mathrm{~W}$ \\
AMAM-AMPM & $-\mathbf{4 0 d B c}$ & $18 \mathrm{~W}$ \\
Volterra & $-\mathbf{4 3 d B c}$ & $18 \mathrm{~W}$ \\
VSV - K-means (10 clusters) & $-47 \mathbf{d B c}$ & $\mathbf{1 8 W}$ \\
VSV - SOM (10 clusters) & $-\mathbf{4 9 . 7 5 d B c}$ & $\mathbf{1 8 W}$ \\
\hline
\end{tabular}

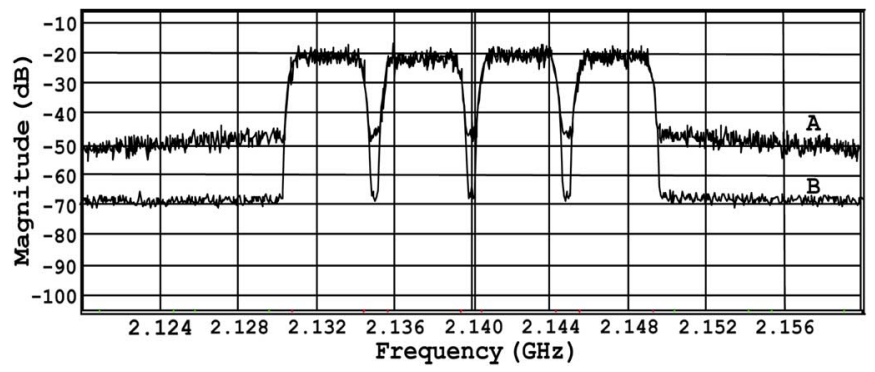

Fig. 3. Illustration of un-linearized spectral plot (A), ACPR $26 \mathrm{dBc}$, superimposed upon linearized spectral plot (B), ACPR $49.5 \mathrm{dBc}$.

imbalance between outphasing paths and amplitude and phase ripple over frequency. The measurement setup uses a pattern generation board paired with a dual transmit chain digital to analog conversion board. Signal capture is performed using a wideband dual channel analog to digital board with quadrature demodulation, the useable bandwidth of the captured signal is $307.2 \mathrm{MHz}$. A wide bandwidth receive path is required to capture the out of band residual outphasing noise, again with a useable bandwidth of at least $307.2 \mathrm{MHz}$. The signal capture was averaged eight times to remove measurement noise that may impact the calculation of the linearization function.

The linearization function is trained in an iterative process utilizing an indirect training technique and validated experimentally in hardware. The performance of the system was determined using a Rhode and Schwarz FSQ spectrum analyzer and multicarrier ACPR measurement, the results of which are illustrated graphically in Fig. 3, the spectrum before and after linearization and presented numerically in Table I.

For linearization the delay factor in the vector quantization function is a 2 tap delay. The individual Volterra series kernels have a 5 th order non-linearity component with 3 memory terms. These optimum parameters were found by sweeping the values across reasonable ranges, non-linear order $\{3,5,7\}$, memory depth $\{2-5\}$, delay taps for generating vector space $\{1-4\}$ and number of segments in the vector space $\{6-15\}$. In the training process, vector quantization training is preformed initially, the results are used to segment the training data and each time series is trained individually. The method of training the time series is a least squares algorithm. For stability of the training algorithm QR decomposition is performed on the Volterra kernel matrix. The predistortion function was trained using five iter- ations, at which point it was determined that the linearization performance was at its maximum and further iterations would not provide improvement. The resulting performance can be seen in Fig. 3. The amplifier meets 3GPP spectral mask requirements for both adjacent and alternative channels of $-45 \mathrm{dBc}$ and $-50 \mathrm{dBc}$ respectively. Table I presents the linearization results comparing AMAM AMPM [11], Volterra series, VSV Volterra - K-means and VSV Volterra - SOM using 3 parameters of the input signal.

\section{CONCLUSION}

A single input-output linearization algorithm can successfully correct a calibrated two path outphasing system, confirming the deviations observed at lower output powers are deterministic and therefore correctable. Utilizing a VSV linearization method with SOM linearization performance for wide band signals was improved. The performance benefits of using a SOM as the clustering algorithm are not cost free. In this letter we have presented a linearization technique for an outphasing amplifier with a signal bandwidth of $20 \mathrm{MHz}$ which meet $3 \mathrm{GPP}$ spectral mask requirements.

\section{ACKNOWLEDGMENT}

The authors would like to thank the staff of NXP Semiconductor, Eindhoven, for providing the outphasing PA.

\section{REFERENCES}

[1] M. Helaoui, S. Boumaiza, and F. M. Ghannouchi, "On the Outphasing Power Amplifier Nonlinearity Analysis and Correction Using Digital Predistortion Technique," in Proc. IEEE Radio Wireless Symp., Orlando, FL, 2008, pp. 751-754.

[2] W. Gerhard and R. Knochel, "Nonlinear Behaviour And Digital Phase Predistortion Of Outphasing Power Amplifiers," in Proc. Microw. Conf. (GeMIC), Hamberg, Germany, 2008, pp. 89-92.

[3] J. Fritzin et al., "Phase Predistortion of a Class-D Outphasing RF Amplifier in $90 \mathrm{~nm}$ CMOS," IEEE Trans. Circuits Syst., vol. 58, no. 10, pp. 642-646, Oct. 2011.

[4] Y. Jung, J. Fritzin, M. Enqvist, and A. Alvandpour, "Least-Squares Phase Predistortion of a $+30 \mathrm{dBm}$ Class-D Outphasing RF PA in 65 nm CMOS," IEEE Trans. Circuits Syst., vol. 60, no. 7, pp. 1915-1928, July. 2013.

[5] J. H. Qureshi et al., "A 90-W Peak Power GaN Outphasing Amplifier With Optimum Input Signal Conditioning," IEEE Trans. Microw. Theory Tech.,, vol. 57, no. 8, pp. 1925-1935, Aug. 2009.

[6] M. P. van der Heijden, M. Acar, J. S. Vromans, and D. A Calvillo-Cortes, "A 19 W high-efficiency wide-band CMOS-GaN class-E Chireix RF outphasing power amplifier," in IEEE MTT-S Int. Dig., Baltimore, MD, 2011, pp. 1-4.

[7] S. Afsardoost, T. Eiksson, and C. Fager, "Digital Predistortion Using a Vector-Switched Model," IEEE Trans. Microw. Theory Tech., vol. 60, no. 4, pp. 1166-1174, 2012.

[8] D. W. Patterson, Artificial Neural Networks - Theory and Applications. Princeton, NJ: Prentice Hall, 1996

[9] F. Bacao, V. Lobo, and M. Painho, "Self-organizing Maps as Substitutes for K-Means Clustering," in Proc. 5th Int. Conf. Comp. Sci., Atlanta, GA, 2005, pp. 476-483.

[10] F. Maiorana, "Performance Improvements of a Kohonen Self Organizing Classification Algorithm on Sparse Data Sets," in Proc. 10th WSEAS Int. Conf. Math. Methods, Comp. Tech. Intell. Syst., Corfu, 2008, pp. 347-352.

[11] J. K. Cavers, "Amplifier linearization using a digital predistorter with fast adaptation and low memory requirements," IEEE Trans. Veh. Technol., vol. 39, no. 4, pp. 374-382, Nov. 1990 